\title{
Research Programs and Plans at the Soreq Applied Research Accelerator Facility - SARAF
}

\author{
Israel Mardor ${ }^{1}$, Dan Berkovits, Shlomi Halfon, Tsviki Hirsh, Yonatan Mishnayot, \\ Ido Silverman, Sergey Vaintraub, Leo Weissman \\ Soreq NRC \\ Yavne 81800, Israel \\ E-mail: mardoresoreq.gov.il
}

Michael Hass, Ish Mukul

The Weizmann Institute of Science

Rehovot 76100, Israel

Ben Ohayon, Michael Paul, Guy Ron, Moshe Tessler

Racah Institute of Physics, The Hebrew University

Jerusalem 91904, Israel

\section{Timo Dickel}

II. Physikalisches Institut, Justus-Liebig-Universität Gießen, Gießen, Germany

GSI Helmholtzzentrum für Schwerionenforschung GmbH, Darmstadt, Germany

The Soreq Applied Research Accelerator Facility (SARAF) is under construction at the Soreq Nuclear Research Center, Yavne, Israel. When completed at the beginning of the next decade, SARAF will be a user facility based on a $40 \mathrm{MeV}, 5 \mathrm{~mA} \mathrm{CW}$ proton/deuteron superconducting linear accelerator. Phase I of SARAF (4 MeV, $2 \mathrm{~mA} \mathrm{CW}$ protons, $5 \mathrm{MeV} 1 \mathrm{~mA}$ pulsed deuterons) is already in operation. By use of a novel liquid lithium jet target (LiLiT), we generated up to $5 \times 10^{10}$ epithermal neutrons/sec, mainly for nuclear astrophysics research of slow neutron capture processes (s-process). We present a survey of research programs and plans at the completed SARAF, which span several disciplines: Precision studies of beyond-Standard-Model effects by trapping light exotic isotopes, such as ${ }^{6} \mathrm{He},{ }^{8} \mathrm{Li}$ and $\mathrm{Ne}$ isotopes, in unprecedented amounts (including meaningful studies already at Phase I); extended nuclear astrophysics research with higher energy neutrons, including generation and studies of exotic neutron-rich isotopes relevant to the rapid ( $\mathrm{r}-$ ) process; high energy neutrons cross section measurements for basic nuclear physics and material science research, including neutron induced radiation damage; neutron based imaging and therapy; and novel radio-pharmaceuticals development and production.

The 26th International Nuclear Physics Conference

11-16 September, 2016

Adelaide, Australia

${ }^{1}$ Speaker 


\section{Introduction}

Soreq Nuclear Research Center (SNRC) is constructing the Soreq Applied Research Accelerator Facility (SARAF), a user facility that will be based on a light ion (protons and deuterons), medium energy (40 MeV) high $\mathrm{CW}$ current (5 mA) accelerator, to be completed at the first half of the next decade. These specifications, with the proper targets, will make SARAF one of the world's most powerful deuteron, proton and fast neutron sources.

SARAF will enable precise measurements of rare phenomena, especially away from the valley of stability, which may provide an excellent probe to our understanding of nuclear physics, the genesis of elements in the universe, and physics beyond the Standard Model of elementary particles. Further plans in SARAF include material research for generation IV fission power reactors and fusion reactors, accelerator-based neutron imaging, and the development of new medical treatments and pharmaceuticals.

Phase I of SARAF (4 MeV, $2 \mathrm{~mA} \mathrm{CW}$ protons, $5 \mathrm{MeV} 1 \mathrm{~mA}$ pulsed deuterons) is already in operation. By use of a novel liquid lithium jet target (LiLiT) [1, 2], we generated up to $5 \times 10^{10}$ epi-thermal neutrons/sec, mainly for nuclear astrophysics research of slow neutron capture processes (s-process) [3] and also for feasibility studies of Boron Neutron Capture Therapy (BNCT) [4]. For an overview of SARAF Phase I and its ongoing and near future plans, see [5, 6] and references therein.

In this paper we describe the research programs and plans at the completed SARAF, including their expected scientific importance and impact.

\section{The Specialty of SARAF}

Irradiation of deuterons on light targets in the range of tens of $\mathrm{MeV}$, which will be available at SARAF, will generate fast neutrons with a forward flux and energy peaked around the beam energy divided by 2.5. It will thus be possible to obtain with SARAF local fluxes of high energy neutrons that are significantly higher than those available at spallation sources. For example, irradiation of lithium by $40 \mathrm{MeV}$ deuterons generates a local neutron flux in the energy range $5-25 \mathrm{MeV}$, which is higher by an order of magnitude than that obtained by irradiation of $1400 \mathrm{MeV}$ protons on tungsten (Fig. 1). Notice that the comparison is per beam particle, so SARAF's advantage is even more significant given its higher beam current.

In Table 1 we compare the expected neutron output of SARAF with the similar upcoming medium energy deuteron projects, SPIRAL-II and IFMIF. From Table 1 we see that the combination of a $5 \mathrm{~mA}$ beam and a liquid lithium target marks the specialty and competitiveness of SARAF, due to its advantageous beam spot size, beam density on target and maximum neutron flux, enabling efficient neutron irradiation of small and rare targets.

In addition to the direct use of the neutrons, we are planning to use them within a twostage target to produce exotic light ions at extremely high rates. The first stage is liquid lithium, and the second, which is irradiated by the neutron flux, is selected based on the specific exotic light ion that one seeks $[5,6,7]$. 


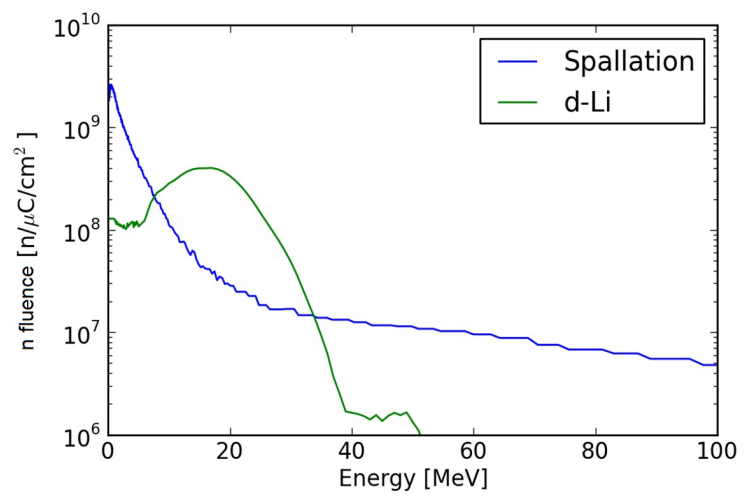

Figure 1: Neutron spectra, simulated for $40 \mathrm{MeV}$ deuterons on $\mathrm{Li}$, and measured for spallation $1400 \mathrm{MeV}$ protons on $\mathrm{W}$ (ISOLDE), $8 \mathrm{~cm}$ downstream of the targets at $0^{\circ}$.

\begin{tabular}{|l|c|c|c|}
\hline \multicolumn{1}{|c|}{ Parameter } & IFMIF & SPIRAL-II & SARAF \\
\hline Reaction (all at $40 \mathrm{MeV})$ & $\mathrm{d}+\mathrm{Li}$ & $\mathrm{d}+\mathrm{C}$ & $\mathrm{d}+\mathrm{Li}$ \\
\hline Projectile range in target $[\mathrm{mm}]$ & 19.1 & 4.3 & 19.1 \\
\hline Maximal beam current $[\mathrm{mA}]$ & $2 \times 125$ & 5 & 5 \\
\hline Beam spot on target $\left[\mathrm{cm}^{2}\right]$ & $\sim 100$ & $\sim 10$ & $\sim 1$ \\
\hline Beam density on target $\left[\mathrm{mA} / \mathrm{cm}^{2}\right]$ & 2.5 & 0.5 & 5 \\
\hline Neutron production over $4 \pi(\mathrm{n} / \mathrm{d})$ & $\sim 0.07$ & $\sim 0.03$ & $\sim 0.07$ \\
\hline Neutron source intensity $(\mathrm{n} / \mathrm{sec})$ & $\sim 10^{17}$ & $\sim 10^{15}$ & $\sim 10^{15}$ \\
\hline $\begin{array}{l}\text { Maximum neutron flux on the back-plate } \\
(0-60 \mathrm{MeV})\left[\mathrm{n} / \mathrm{sec}^{2} \mathrm{~cm}^{2}\right]\end{array}$ & $\sim 10^{15}$ & $\sim 10^{14}$ & $\sim 5 \times 10^{14}$ \\
\hline$<$ En> on the back-plate $[\mathrm{MeV}]$ & $\sim 10$ & $\sim 12$ & $\sim 10$ \\
\hline
\end{tabular}

Table 1: Anticipated neutron output of SARAF, compared to SPIRAL-II and IFMIF [3].

\section{Research Programs and Plans at SARAF}

\subsection{Beyond Standard Model Physics and Light Exotic Isotopes research}

Searches for physics beyond the Standard Model (SM) are carried out both at the high energy frontier, attained at the most powerful particle colliders, as well as at the high precision frontier, looking for deviations from SM predictions in low background environments, where high sensitivities to small effects can often be achieved [9].

In SARAF, we will explore the precision frontier by generating ultra-pure samples of exotic nuclei and trapping them. We will produce light exotic nuclei such as ${ }^{6} \mathrm{He},{ }^{8} \mathrm{Li}$ and $\mathrm{Ne}$ isotopes in unprecedented amounts [10], trap them, and measure their $\beta$-decay parameters with ultra-high precision [11]. These decay parameters (angular correlations, polarization asymmetry, life-time, transition energy and more) may provide signatures for beyond-SM phenomena, including scalar and tensor transitions, time-reversal violation, right handed currents and neutrinos, and deviation from CKM quark mixing matrix unitarity [9]. In this respect, $\mathrm{Ne}$ isotopes are attractive as they are among the least precisely measured of the isotopes used in the search for beyond SM physics (Fig. 2). 


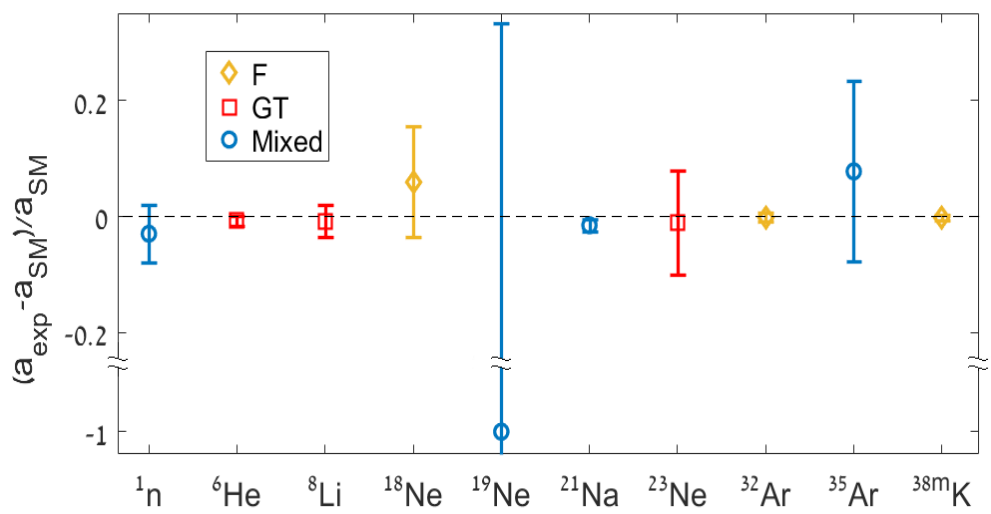

Figure 2: Experimentally obtained $\beta-v$ correlation coefficient compared to standard-model value for various isotopes. The most precise value was selected for each isotope.

The first SARAF measurements in this context will be of $\beta-\nu$ correlations of ${ }^{6} \mathrm{He}$ and $\mathrm{Ne}$ isotopes, in search of tensor and scalar components of the $\beta$-decay. For ${ }^{6} \mathrm{He}$ we will use an Electrostatic Ion Beam Trap (EIBT) and for Ne a Magneto-Optical Trap (MOT) (Fig. 3).
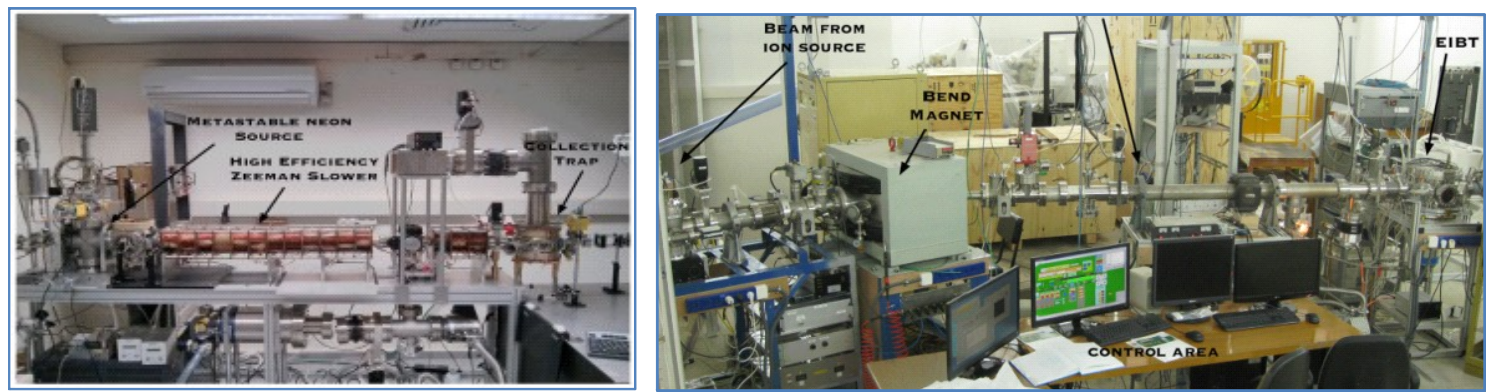

Figure 3: The MOT setup at Hebrew University at Jerusalem (left) and the EIBT setup at Weizmann Institute (right) [11], both constructed for use at SARAF.

The varied selection of radiactive light isotopes at SARAF will enable systematic research of nuclei and unbound states towards the proton drip line up to $Z \sim 11$, via $(\mathrm{p}, \mathrm{xn})$ and $(\mathrm{d}, \mathrm{xn})$, and the neutron drip up to $Z \sim 4$ via $(n, \gamma),(d, p),(n, p)$ and $(n, d)$. In a future SARAF upgrade, a distinct spectrum of neutrinos and anti-neutrinos, from ${ }^{6} \mathrm{He},{ }^{8} \mathrm{Li}$ and ${ }^{18} \mathrm{Ne}$ decays, may be detected at rates of $10^{-3}-10^{-4} \mathrm{v} / \mathrm{sec}$, depending on specific detector configurations, enabling direct measurements of $\mathrm{CP}$ properties in the neutrino sector.

\subsection{Nuclear Astrophysics and Neutron-Rich Fission Fragment Research}

As described in [3], studies of the astrophysical s-process via epithermal neutron capture reactions constitute the main research program of SARAF Phase I. Certainly these studies may continue in the completed SARAF, and in addition, the increased flux of neutrons at higher energies will enable further studies of astrophysical phenomena.

For example, the reactions that destroy ${ }^{26} \mathrm{Al}$ in massive stars $\left({ }^{26} \mathrm{Al}(\mathrm{n}, \mathrm{p}){ }^{26} \mathrm{Mg}\right.$ and ${ }^{26} \mathrm{Al}(\mathrm{n}, \alpha)^{23} \mathrm{Na}$ ) at temperatures up to $\sim 10 \mathrm{GK}$ (equivalent to $\mathrm{E}_{\mathrm{n}} \sim 900 \mathrm{keV}$ ) have astrophysical significance [12]. Nevertheless, the available data are sparse, largely because ${ }^{26} \mathrm{Al}$ is radioactive. Fig. 4 shows the experimental reaction rates for ${ }^{26} \mathrm{Al}(\mathrm{n}, \alpha)^{23} \mathrm{Na}$, where the results of Koehler and 
De Smet are from direct neutron reactions (up to $\mathrm{E}_{\mathrm{n}} \sim 100 \mathrm{keV}$ ) and the higher temperature / energy results of Skelton are deduced from ${ }^{23} \mathrm{Na}(\alpha, \mathrm{n})^{26} \mathrm{Al}$ (see [12] and references therein).

The high neutron flux at SARAF (Table 1) will enable direct neutron measurements at all relevant energies even on rare, radioactive and extremely small targets such as ${ }^{26} \mathrm{Al}$.

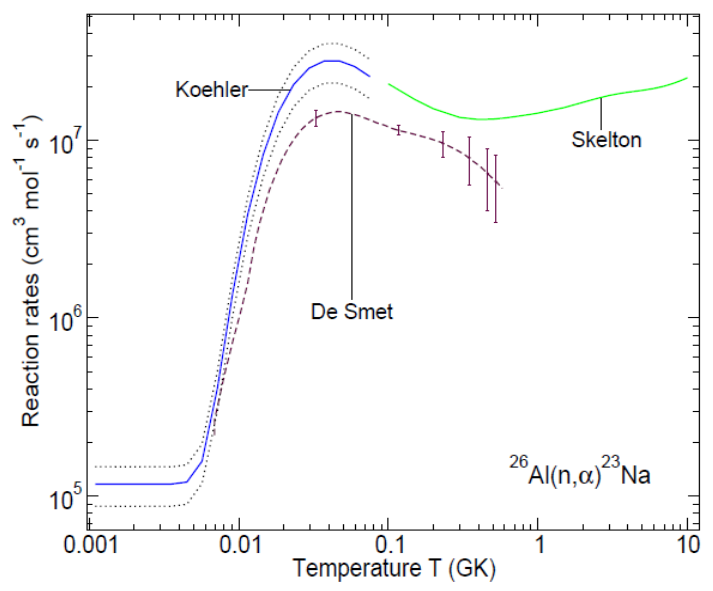

Figure 4: Reaction rate of ${ }^{26} \mathrm{Al}(\mathrm{n}, \alpha)^{23} \mathrm{Na}$ as a function of temperature. Data sources are discussed in the text and in Ref. 12. Figure reprinted with permission from Ref. 12.

Detailed studies of neutron-rich nuclides provide crucial input to the study of the astrophysical r-process [13]. Such nuclides may be generated at SARAF via neutron induced fission on thin ${ }^{238} \mathrm{U}$ targets [14]. This reaction is optimal for generation of neutron-rich nuclides at $E_{\mathrm{n}} \sim 10 \mathrm{MeV}$ [15]. A prototype for generation and extraction of high energy neutron induced fission fragments has been demonstrated at Jyvaskyla [15]. A possible setup of such a facility at SARAF is depicted in Figure 5, inspired by the Ion Catcher at the end of the Fragment Separator (FRS) at GSI [16].

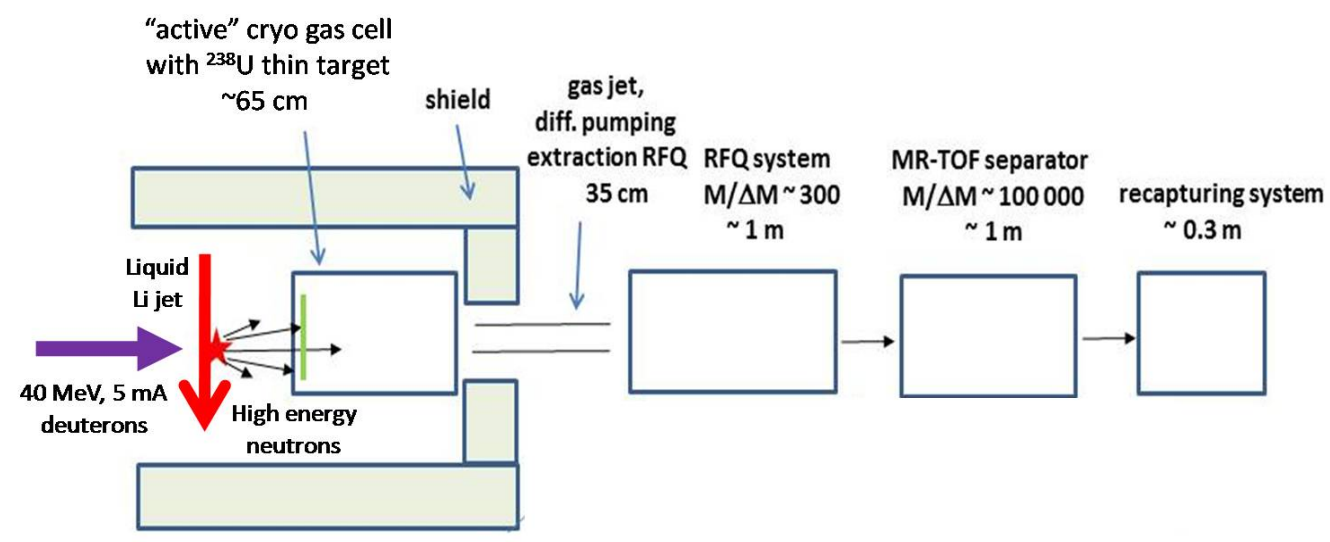

Figure 5: Schematic layout of a neutron-induced fission fragment generation, extraction, identification and research facility at SARAF. Length may be $\sim 4 \mathrm{~m}$, including the Li target.

Given the expected flux of $<\mathrm{En}>\sim 10 \mathrm{MeV}$ neutrons (Table 1), the ${ }^{238} \mathrm{U}$ neutron-induced fission cross section at this neutron energy ( $\sim 1$ barn [17]), and a 10 micron thick ${ }^{238} \mathrm{U}$ target with area in order of $\sim \mathrm{cm}^{2}$, the estimated fission yield at SARAF is $\sim 5 \times 10^{9}$ fissions/sec.

Based on fission branching ratios [18], target escape fraction $(\sim 0.5$ [19]), and an overall extraction and identification efficiency in the instrumentation of Fig. $5(\sim 0.15$ [14]), we estimated the rates for recaptured neutron-rich fission fragments at SARAF. In Figure 6 we 
present the ratios between the estimated SARAF rates (recaptured) and FRIB rates (reaccelerated) [20], for the nuclides whose SARAF rate is higher. From Figure 6 it is clear that SARAF will provide world competitive rates at the fission fragment region.

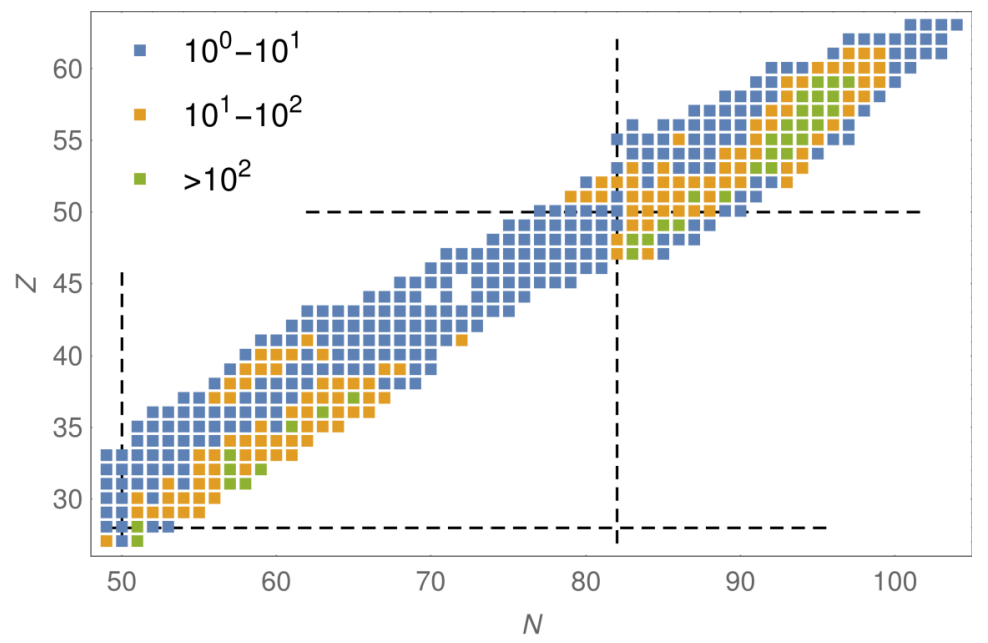

Figure 6: Ratios between neutron-rich nuclide rates (nuclides/sec) at SARAF (recaptured) and FRIB (reaccelerated), for the nuclides whose SARAF rate is higher.

\subsection{High Energy neutron-induced cross sections and material research}

Only limited data exist for high-energy neutron induced reactions, usually at discrete points, and frequently inconsistent with theoretical models, for example in neutron-deuteron breakup reactions [21] and neutron scattering from ${ }^{28} \mathrm{Si}$ and ${ }^{32} \mathrm{~S}$ [22]. Further, high-energy neutron-induced calibration data is needed for rare event searches, such as for dark matter [23] and neutrino-less double- $\beta$ decay [24].

In addition, material testing via irradiation in high energy and high flux neutrons are of great interest for accelerator driven systems (ADS), Generation-IV fission reactors and future fusion demonstrators. For fusion reactors, one of the main risks is high energy ( $14 \mathrm{MeV})$ neutron induced radiation damage due to $\mathrm{d}+\mathrm{t}$ fusion.

SARAF will enable extensive research with high energy neutrons, with a variable energy peak and a maximal value of $\sim 50 \mathrm{MeV}$, which could cope with the challenges described above. In particular, the local neutron flux at SARAF, expected to be only a factor of 2 less than IFMIF (Table 1), may place it as an intermediate facility for irradiation of miniaturized material samples (e.g., $0.5 \mathrm{~mm}$ beryllium pebbles, which compose the ITER multiplier blankets [25]).

\subsection{Neutron Based Imaging}

One of the goals of SARAF is to demonstrate that activities of a typical research reactor can be carried out in medium energy high current ion accelerators [26]. For that purpose, a thermal neutron imager was designed for SARAF, which includes a beryllium target, amplifier and a $\mathrm{D}_{2} \mathrm{O}$ moderator [27]. The designed cooling concept of the target, using mini- and microchannels, has been proven experimentally to sustain $\sim 1 \mathrm{~kW} / \mathrm{cm}^{2}$ [28]. There is currently an effort to generate an updated design of a thermal neutron source based on a liquid lithium target. 
In addition to thermal neutrons, with an accelerator one may envision imaging with fast neutrons, which enable better contrast over broad densities and atomic numbers. By using a d-t neutron generator, the effects of different types and materials of collimators were studied [29].

\subsection{Development of New Radiopharmaceuticals}

In its 2009 report [30], the NSAC Isotopes Subcommittee stated that a $40 \mathrm{MeV}$, variableenergy, multi-particle is the optimal accelerator for radiopharmaceutical production. The completed SARAF specifications precisely match these requirements. In particular, the availability of deuteron beams will enable high yield production of neutron deficient isotopes, since $(\mathrm{d}, 2 \mathrm{n})$ is more prolific than $(\mathrm{p}, \mathrm{n})$ for some $\mathrm{A}>100$ elements. Further, $(\mathrm{d}, \mathrm{p})$ can generate neutron rich isotopes, providing an alternative for $(\mathrm{n}, \gamma)$ at nuclear reactors [31].

\section{Summary}

SARAF is a high power accelerator based user facility under construction at Soreq NRC. Phase I is completed and operational. The full SARAF facility is mostly funded, and foreseen to commence operations towards the first half of the next decade, with world competitive $p, d$, and $\mathrm{n}$ fluxes, generating especially high rates in the light exotic and fission fragment regions.

In this paper we described the currently envisioned research programs and plans at the completed SARAF. We look forward to additional proposals and collaborations.

\section{References}

[1] S. Halfon et al., High-power liquid-lithium jet target for neutron production, Rev. Sci. Inst. 84, 123507 (2013)

[2] S. Halfon et al., Note: Proton irradiation at kilowatt-power and neutron production from a freesurface liquid-lithium target, Rev. Sci. Inst. 85, 056105 (2014)

[3] M. Tessler et al., Stellar 30-keV neutron capture in ${ }^{94,96} \mathrm{Zr}$ and the ${ }^{90} \mathrm{Zr}(\gamma, n)^{89} \mathrm{Zr}$ photonuclear reaction with a high-power liquid-lithium target, Phys. Lett. B 751, 418 (2015)

[4] S. Halfon et al., Demonstration of a high-intensity neutron source based on a liquid-lithium target for Accelerator based Boron Neutron Capture Therapy, Appl. Rad. and Isot. 106, 57 (2015)

[5] I. Silverman et al., Scientific opportunities at SARAF with a liquid lithium jet target neutron source, Proceedings of INTDS 2016, Submitted to Physics Procedia, 2017

[6] I. Mardor, D. Berkovits (2015), The Soreq Applied Research Accelerator Facility (SARAF), Nuclear Physics News, 25:1, 16-22

[7] T. Stora et al. (2012), A high intensity ${ }^{6} \mathrm{He}$ beam for the $\beta$-beam neutrino oscillation facility, EPL 98, 32001

[8] D. Ridikas et al., Internal Report DSM/DAPNIA/SPhN, CEA Saclay (2003)

[9] O. Naviliat-Cuncic, M. González-Alonso (2013), Prospects for precision measurements in nuclear $\beta$ decay in the LHC era, Annalen Der Physik, 525: 600-619 [hep-ph/1304 . 1759]

[10] M. Hass et el., Light radio-isotopes for nuclear astrophysics and neutrino physics, Journal of Physics G35, 014042 (2008); T. Y. Hirsh et al., Towards an intense radioactive ${ }^{8}$ Li beam at SARAF Phase I, Journal of Physics: Conference Series 337 (2012) 012010 
[11] O. Aviv, et al., Beta decay measurements from ${ }^{6} \mathrm{He}$ using an electrostatic ion beam trap, Journal of Physics: Conference Series 337 (2012) 012020

[12] B. Oginni et al., Theoretical Evaluation of the Reaction Rates for ${ }^{26} \mathrm{Al}(n, p){ }^{26} \mathrm{Mg}$ and ${ }^{26} \mathrm{Al}(n, \alpha)^{23} \mathrm{Na}$, Phys. Rev. C83 025802 (2011), DOI:https://doi.org/10.1103/PhysRevC.83.025802

[13] M. Arnould et al., The r-process of stellar nucleosynthesis: Astrophysics and nuclear physics achievements and mysteries, Physics Reports, 450 (2007) 97 - 213

[14] L. Weissman and M. Paul, Neutron-rich radioactive-ion production at SARAF phase-II, SNRC internal report, Soreq Nuclear Research Center, October 2013

[15] D. Gorelov et al., Developments for neutron-induced fission at IGISOL-4, Nucl. Instr. and Meth. B376 (2016) $46-51$

[16] T. Dickel et al., A high-performance multiple-reflection time-of-flight mass spectrometer and isobar separator for the research with exotic nuclei, Nucl. Instr. and Meth. A777 (2015) 172 - 188

[17] N. Otuka et al., Towards a More Complete and Accurate Experimental Nuclear Reaction Data Library (EXFOR): International Collaboration Between Nuclear Reaction Data Centres (NRDC), Nuclear Data Sheets120 (2014) 272 - 276

[18] http://wwwndc.jaea.go.jp/cgi-bin/FPYfig?iso=nU238\&eng=e2

[19] S. Kahn et al., Energy Distributions of Fission Fragments from Uranium Dioxide Films, Nucl. Sci. and Eng. 23 (1965) $8-20$

[20] https://groups.nscl.msu.edu/frib/rates/fribrates.html

[21] A. H. Couture et al., Cross-section measurements of ${ }^{2} H(n, n p) n$ in symmetric star configurations, Phys. Rev. C 85, 054004 (2012)

[22] M. A. Al-Ohali et al., Neutron scattering from ${ }^{28} \mathrm{Si}$ and ${ }^{32} \mathrm{~S}$ from 8.0 to $18.9 \mathrm{MeV}$, dispersive optical model analyses, and ground-state correlations, Phys. Rev. C 86, 034603 (2012)

[23] S. MacMullin et al., Measurement of the elastic scattering cross section of neutrons from argon and neon, Phys. Rev. C 87, 054613 (2013)

[24] C. Bhatia et al, ${ }^{136} \mathrm{Xe}(n, 2 n){ }^{135} \mathrm{Xe}$ cross section between 9 and $15 \mathrm{MeV}$, Phys. Rev. C 87, 011601(R) (2013)

[25] E. Rabaglino, Helium and tritium in neutron irradiated beryllium, FZKA 6939, Karlsruhe (2004)

[26] T. Feder, Seeking to bridge Europe's impending neutron gap, Physics Today 69(3), 25 (2016)

[27] K. Lavie, M.Sc. Thesis, Design of a compact thermal neutron source for radiography based on $S A R A F$, Ben-Gurion University, Israel (2005)

[28] H. Hirshfeld et al., High heat flux cooling of accelerator targets with micro-channels, Nucl. Inst. Meth. A562, 903 (2006)

[29] I. Sabo-Napadensky et al., Research and development of a dedicated collimator for $14.2 \mathrm{MeV}$ fast neutrons for imaging using a D-T generator, JINST 7, C06005 (2012)

[30] D. Geesaman et al., Reaching for the horizon: The 2015 long range plan for nuclear science, U.S. Department of Energy, 2015

[31] A. Hermanne et al., Production of medically relevant radionuclides with medium energy deuterons, International Conference on Nuclear Data for Science and Technology 2007 